\title{
Terpenoids from Clerodendrum formicarum Gürke (Lamiaceae) of Cameroon
}

\author{
Muhammad Shaiq Ali ${ }^{\mathrm{a}}$, Zeeshan Ahmed ${ }^{\mathrm{a}}$, Joseph Ngoupayo ${ }^{\mathrm{b}}$, and Muhammad Imran Ali ${ }^{\mathrm{a}}$ \\ ${ }^{a}$ H. E. J. Research Institute of Chemistry, International Center for Chemical and Biological Sciences, \\ University of Karachi, Karachi, Pakistan \\ b Department of Pharmacy and African Pharmacotherapeutics, Faculty of Medicine and Biological \\ Sciences, University of Yaounde I, P. O. Box 1364, Yaounde, Cameroon
}

Reprint requests to Muhammad Shaiq Ali. E-mail: shaiq303@hotmail.com

Z. Naturforsch. 2010, 65b, 521-524; received November 5, 2009

\begin{abstract}
The ethanolic extract of the leaves of Clerodendrum formicarum, a Lameacious plant of Cameroon, afforded a new abeo-abietane diterpenoid named formidiol (1) together with some known diand triterpenoids. They include 12,16-epoxy-11,14-dihydroxy-6-methoxy-17(15 $\rightarrow 16)$-abeo-abieta5,8,11,13,15-pentanene-3,7-dione (2), trans-phytol, friedelin and friedlan-3 $\beta$-ol. Structures of all the isolated constituents have been characterized with the aid of 1D NMR spectroscopy while the structure of a new metabolite was elucidated via 2D NMR spectroscopic techniques.
\end{abstract}

Key words: Formidiol, abeo-Abietane, Leaves, Clerodendrum formicarum, Lamiaceae, Characterization, Spectroscopy

\section{Introduction}

Clerodendrum L. of the family Lamiaceae is a very large and diverse genus, and five hundred and eighty species including small trees, shrubs and herbs distributed in Asia, Australia, Africa, and America. The genus has been used as medicines specifically in India, China, Thailand, Korea, and Japan for the treatment of various life threatening diseases such as syphilis, typhoid, cancer, jaundice, and hypertension. Various species of the genus Clerodendrum are known to possess potent bioactivities. Hexane extracts of $C$. colebrookianum show strong antibacterial activities against Staphylococcus aureus, S. haemolyticus, E. coli, Pseudomonas aeruginosa [1]. The alcoholic extracts of $C$. phlomidis exhibit antimalarial activity against Plasmodium falciparum [2]. CNS-related activities were also observed in $C$. phlomidis showing tranquillizing, CNS-depressant and muscle-relaxant properties in experimental mice and rats [3]. A decoction of C. phlomidis (whole plant) has been reported to have antidiabetic activity [4]. C. inerme has been used as an antioxidant drug in various indigenous systems of medicines [5]. C. bungei show antitumor activity in hepatic cells of mice [6].

The major chemical components reported from the genus Clerodendrum are iridoids [7], iridoid glucosides [8], steroids [9], steroidal glycosides [10], ter-<smiles>CC(=O)C1=C(C)C2=CC(=O)c3c(c(C)c4oc(C)cc4c3O)[C@@]2(C)CC1</smiles>

penes [11], flavonoids [12], flavonoid glycosides [13], chalcone glycosides [14], and macrocyclic alkaloids [15]. The present communication describes the isolation and characterization of a new abeo-abietane diterpenoid named formidiol (1) together with some known di- and triterpenoids from the leaves of Clerodendrum formicarum, collected from Obili-Yaounde (Cameroon).

\section{Results and Discussion}

The ethanolic extract of the leaves of $C$. formicarum afforded $\mathbf{1}$ as a red amorphous powder. The IR spectrum of 1 displayed three prominent absorptions. An absorption at $3697 \mathrm{~cm}^{-1}$ is due to the hydroxyl function/s in the molecule while the remain- 


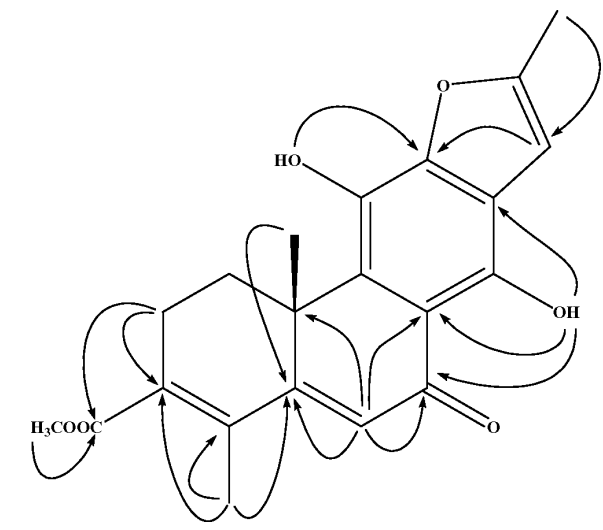

Fig. 1. Important HMBC connectivities in $\mathbf{1}$.

ing two prominent bands at 1615 and $1721 \mathrm{~cm}^{-1}$ attest for an $\alpha, \beta$-unsaturated ketone and an ester carbonyl function, respectively. The molecular ion peak observed in the EIMS at $m / z=368$ and the formula associated with this peak was found as $\mathrm{C}_{21} \mathrm{H}_{20} \mathrm{O}_{6}$ in the high-resolution mass spectrum showing the presence of twelve degrees of unsaturation in the molecule. A significant fragment at $m / z=308$ is due to the loss of a methyl formate molecule from the molecule ion peak confirming the presence of a formate moiety in the molecule.

The ${ }^{1} \mathrm{H}$ NMR spectrum of $\mathbf{1}$ was very simple with only a few signals due to the fact that most of the carbon atoms in the molecule are quaternary in nature. The proton spectrum displayed four methyl singlets at $\delta=2.45$ (H-17), 2.18 (H-19), 1.55 (H-20), and $\delta=3.80$, the latter being due to the methoxyl moiety. The two olefinic protons appeared as singlets at $\delta=6.47$ and 6.60 assigned to $\mathrm{H}-6$ and $\mathrm{H}-15$, respectively. The hydroxyl protons resonated at $\delta=5.20$ (HO-11) and 13.83 (HO-14). The carbon spectrum of 1 showed altogether 21 carbon signals which were further sorted out with the aid of DEPT experiments into four methyls, two methylenes, and two methines with the remaining ones for quaternary carbons. The two methyls directly attached to olefinic quaternarycarbons resonated at $\delta=14.0(\mathrm{C}-17)$ and $17.2(\mathrm{C}-19)$ while the methoxyl group appeared at $\delta=52.0$. Signals at $\delta=122.8$ and 101.6 attested for C-6 and C-15, respectively. An $\alpha, \beta$-unsaturated ketonic carbon appeared at $\delta=190.9$ and the ester carbonyl function at $\delta=169.1$. The olefinic-quaternary carbon to which the formate moiety is attached was observed at $\delta=132.9$. Signals of hydroxyl-bearing carbons appeared at $\delta=$ 131.4 (C-11) and 117.3 (C-14). A complete picture of
Table $1 .{ }^{13} \mathrm{C}-\mathrm{NMR}$ data of $\mathbf{1}$ and $\mathbf{2}$ ( $\delta_{C}$ in ppm; multiplicity in parentheses; solvent $\mathrm{CDCl}_{3}$ ).

\begin{tabular}{crr}
\hline Position & \multicolumn{1}{c}{$\mathbf{1}$} & \multicolumn{1}{c}{$[16]$} \\
\hline 1 & $25.0(\mathrm{t})$ & $27.2(\mathrm{t})$ \\
2 & $29.2(\mathrm{t})$ & $33.1(\mathrm{t})$ \\
3 & $132.9(\mathrm{~s})$ & $214.0(\mathrm{~s})$ \\
4 & $128.8(\mathrm{~s})$ & $49.5(\mathrm{~s})$ \\
5 & $163.4(\mathrm{~s})$ & $155.7(\mathrm{~s})$ \\
6 & $122.8(\mathrm{~d})$ & $146.1(\mathrm{~s})$ \\
7 & $190.9(\mathrm{~s})$ & $186.8(\mathrm{~s})$ \\
8 & $109.4(\mathrm{~s})$ & $109.3(\mathrm{~s})$ \\
9 & $136.0(\mathrm{~s})$ & $127.5(\mathrm{~s})$ \\
10 & $39.0(\mathrm{~s})$ & $41.5(\mathrm{~s})$ \\
11 & $131.4(\mathrm{~s})$ & $130.8(\mathrm{~s})$ \\
12 & $148.8(\mathrm{~s})$ & $148.6(\mathrm{~s})$ \\
13 & $117.3(\mathrm{~s})$ & $117.4(\mathrm{~s})$ \\
14 & $151.8(\mathrm{~s})$ & $151.7(\mathrm{~s})$ \\
15 & $101.6(\mathrm{~d})$ & $101.5(\mathrm{~d})$ \\
16 & $155.1(\mathrm{~s})$ & $155.4(\mathrm{~s})$ \\
17 & $14.0(\mathrm{q})$ & $14.0(\mathrm{q})$ \\
18 & $169.1(\mathrm{~s})$ & $25.9(\mathrm{q})$ \\
19 & $17.2(\mathrm{q})$ & $22.5(\mathrm{q})$ \\
20 & $21.6(\mathrm{q})$ & $20.5(\mathrm{q})$ \\
$\mathrm{OCH}_{3}$ & $52.0(\mathrm{q})$ & $60.1(\mathrm{q})$ \\
\hline
\end{tabular}<smiles>COC1=C2C(C)(C)C(=O)CC[C@]2(C)c2c(c(O)c3cc(F)oc3c2O)C1=O</smiles>

the carbon spectrum of $\mathbf{1}$ is given in Table 1. Assignments of various protons and their associated carbons in the NMR spectra of $\mathbf{1}$ were correlated via HMQC experiments and cross-checked via HMBC connectivities (Fig. 1).

A known abietane diterpenoid (2) which was previously isolated by Barros et al. from Aegiphila lhotzkiana [16] was also isolated by us from the same source. From a comparative NMR-spectral analysis of $\mathbf{1}$ and $\mathbf{2}$ (Table 1) it was concluded that $\mathbf{1}$ is also a diterpenoid of the same class except for a methyl (Me-18) migrated from C-4 to C-3 (abeoabietane) [17]. Abietane [18] and abeo-abietane diterpenoids $[18,19]$ have already been isolated and re- 
ported from various species of the genus Clerodendrum.

On the basis of the above spectral information, the structure of the compound discussed above is elucidated as $\mathbf{1}$ and named formidiol. This compound is a new addition in the series of natural abeo-abietane diterpenoids.

In addition to formidiol (1) and 12,16-epoxy$11,14$-dihydroxy-6-methoxy-17(15 $\rightarrow 16)$-abeo-abieta5,8,11,13,15-pentanene-3,7-dione (2) [16], some more known di- and triterpenoids have also been obtained form the same source. They include trans-phytol, 3 [20], friedelin, 4 [21] and friedlan-3 $\beta$-ol, 5 [22]. Their spectral data are given in the Experimental Section.

\section{Experimental Section}

\section{General}

The melting points were recorded in glass capillary tubes using a Büchi 535 melting point apparatus and are uncorrected. Optical rotation was measured on a Jasco DIP-360 (Japan Spectroscopic Co. Ltd., Tokyo, Japan) digital polarimeter. The IR spectrum was recorded on a Shimadzu IR-460 instrument. The ${ }^{1} \mathrm{H}$ - and ${ }^{13} \mathrm{C}$ NMR spectra were recorded at $600 / 500$ and $150 / 125 \mathrm{MHz}$, respectively, on Bruker AM 600 and AM 500 spectrometers using TMS as an internal standard. The EI mass spectrum was scanned on a Jeol-JMS HX-110 mass spectrometer.

\section{Collection and identification}

The leaves of $C$. formicarum were collected in June 2008, from Obili-Yaounde, Cameroon and identified by Mr. Nana Victor of National Herbarium of Yaounde, Cameroon, where a voucher specimen has been deposited (Herbarium \# HNC-13658).

\section{Extraction, isolation and characterization}

The collected leaves were dried under shade for a week. The dried and powered material $(6.0 \mathrm{~kg})$ was then soaked in ethanol (12 L) for six days. The resulting extract was concentrated by means of evaporation under vacuum distillation (84.5 g) and subjected to silica gel column chromatography using hexane, hexane/ethyl acetate, ethyl acetate, and ethyl acetate/methanol as mobile phase.

\section{Formidiol (1)}

Fractions eluted with $10 \%$ ethyl acetate in hexane afforded 1 as a red amorphous powder $(4.5 \mathrm{mg})$. - UV $\left(\mathrm{CHCl}_{3}\right): \lambda_{\max }(\log \varepsilon)=291.5(3.76) \mathrm{nm} .-[\alpha]_{\mathrm{D}}^{20}=+76.0$ $\left(c=0.78, \mathrm{CHCl}_{3}\right) .-\mathrm{IR}\left(\mathrm{CHCl}_{3}\right): v=3697(\mathrm{OH}), 2925$ $(=\mathrm{CH}), 1721(\mathrm{C}=\mathrm{O}), 1615(\alpha, \beta$-unsaturated ketone $), 1208$ (C-O) $\mathrm{cm}^{-1}$. - MS (EI, $\left.70 \mathrm{eV}\right): \mathrm{m} / z=368[\mathrm{M}]^{+}, 353$ $\left[\mathrm{M}-\mathrm{CH}_{3}\right]^{+}, 308\left[\mathrm{M}-\mathrm{H}_{3} \mathrm{COOCH}\right]^{+}$. - HRMS (EI): $\mathrm{m} / \mathrm{z}=$ 368.1256 (calcd. 368.1260 for $\mathrm{C}_{21} \mathrm{H}_{20} \mathrm{O}_{6}$ ), 353.1082 (calcd. 353.1025 for $\mathrm{C}_{20} \mathrm{H}_{17} \mathrm{O}_{6}$ ), 308.1014 (calcd. 308.1049 for $\left.\mathrm{C}_{19} \mathrm{H}_{16} \mathrm{O}_{4}\right) .-{ }^{1} \mathrm{H}$ NMR (600 MHz, $\mathrm{CDCl}_{3}, 29^{\circ} \mathrm{C}$, TMS): $\delta=13.83(1 \mathrm{H}, \mathrm{s}, \mathrm{HO}-14), 6.60(1 \mathrm{H}, \mathrm{s}, \mathrm{H}-15), 5.20(1 \mathrm{H}, \mathrm{s}$, HO-11), 6.47 (1H, s, H-6), 3.35 (1H, m, H-2 $\beta$ ), 2.45 (3H, s, H-17), 2.18 (3H, s, H-19), $1.62(1 \mathrm{H}, \mathrm{m}, \mathrm{H}-2 \alpha), 1.55(3 \mathrm{H}, \mathrm{s}$, $\mathrm{H}-20), 3.80\left(3 \mathrm{H}, \mathrm{s}, \mathrm{OCH}_{3}\right) .-{ }^{13} \mathrm{C} \mathrm{NMR}\left(150 \mathrm{MHz}, \mathrm{CDCl}_{3}\right.$, $29^{\circ} \mathrm{C}$, TMS): see Table 1. - HMBC: see Fig. 1.

12,16-Epoxy-11,14-dihydroxy-6-methoxy-17(15 $\rightarrow 16)$ abeo-abieta-5,8,11,13,15-pentanene-3,7-dione (2) [16]

Upon further elution with the same polarity, compound 2 was obtained as a yellow powder $(5.0 \mathrm{mg})$. $-\mathrm{M} . \mathrm{p}$. = $245{ }^{\circ} \mathrm{C} .-\mathrm{IR}\left(\mathrm{CHCl}_{3}\right) v=3350(\mathrm{OH}), 1710(\mathrm{C}=\mathrm{O}), 1640$ $\left(\alpha, \beta\right.$-unsaturated ketone) $\mathrm{cm}^{-1}$. - MS (EI, $\left.70 \mathrm{eV}\right): \mathrm{m} / z=370$ $[\mathrm{M}]^{+}, 355$. - HRMS (EI): $m / z=370.1397$ (calcd. 370.1416 for $\left.\mathrm{C}_{21} \mathrm{H}_{22} \mathrm{O}_{6}\right) .-{ }^{1} \mathrm{H}$ NMR (600 MHz, $\mathrm{CDCl}_{3}, 29^{\circ} \mathrm{C}$, TMS): $\delta=13.60(1 \mathrm{H}, \mathrm{s}, \mathrm{HO}-14), 6.49(1 \mathrm{H}, \mathrm{s}, \mathrm{H}-15), 5.22(1 \mathrm{H}$, s HO-11), $3.90\left(3 \mathrm{H}, \mathrm{s}, \mathrm{OCH}_{3}\right), 3.38(1 \mathrm{H}, \mathrm{dt}, J=13.8 \mathrm{~Hz}$, $\mathrm{H}-1 \beta), 2.75$ (2H, m, H-2), 2.45 (3H, d, $J=1.2 \mathrm{~Hz}, \mathrm{H}-17)$, 1.55 (3H, s, H-19), 1.50 (3H, s, H-18), 1.47 (3H, s, H-20). ${ }^{13} \mathrm{C}$ NMR (150 MHz, $\mathrm{CDCl}_{3}, 2{ }^{\circ} \mathrm{C}$, TMS): see Table 1.

\section{trans-Phytol (3) [20]}

Elution with $0.5 \%$ ethyl acetate in hexane, afforded compound 3 as a light mobile oil $(20.0 \mathrm{mg})$. - MS (EI, $70 \mathrm{eV})$ : $m / z=296[\mathrm{M}]^{+} .-{ }^{1} \mathrm{H}$ NMR $\left(500 \mathrm{MHz}, \mathrm{CDCl}_{3}, 29{ }^{\circ} \mathrm{C}\right.$, TMS): $\delta=5.38(1 \mathrm{H}$, br.t, $J=6.9 \mathrm{~Hz}, \mathrm{H}-2), 4.13(2 \mathrm{H}, \mathrm{d}$, $J=6.9 \mathrm{~Hz}, \mathrm{H}-1), 1.64(3 \mathrm{H}, \mathrm{s}, \mathrm{H}-3 \mathrm{a}), 0.81(6 \mathrm{H}, \mathrm{d}, J=6.5 \mathrm{~Hz}$, H-7 $\left.{ }^{\mathrm{a}} \& \mathrm{H}-11\right), 0.84\left(6 \mathrm{H}, \mathrm{d}, J=6.6 \mathrm{~Hz}, \mathrm{H}-15^{\mathrm{a}} \& \mathrm{H}-16\right)$. ${ }^{13} \mathrm{C}$ NMR $\left(125 \mathrm{MHz}, \mathrm{CDCl}_{3}, 29{ }^{\circ} \mathrm{C}, \mathrm{TMS}\right): \delta=59.4(\mathrm{C}-1)$, 123.3 (C-2), 140.3 (C-3), 16.2 (C-3a), 39.9 (C-4), 25.7 (C-5), 36.8 (C-6), 32.8 (C-7), 19.7 (C-7a \& 11a), 37.4 (C-8), 24.5 (C-9), 37.3 (C-10), 32.7 (C-11), 37.3 (C-12), 24.8 (C-13), 39.4 (C-14), 28.0 (C-15), 22.6 (C-15a), 22.7 (C-16).

\section{Friedelin (4) [21]}

Elution with $1 \%$ ethyl acetate in hexane afforded compound 4 as colorless needles $(6.5 \mathrm{mg})$. - M.p. $=259$ $261{ }^{\circ} \mathrm{C}$. - IR $\left(\mathrm{CHCl}_{3}\right): v=1720(\mathrm{C}=\mathrm{O}) \mathrm{cm}^{-1}$. - MS (EI, $70 \mathrm{eV}): \mathrm{m} / \mathrm{z}=426[\mathrm{M}]^{+}, 411,314,302,287,273,257,232$, 218, 205, 163, 123. - HRMS (EI): $\mathrm{m} / z=426.3821$ (calcd. 426.3862 for $\left.\mathrm{C}_{30} \mathrm{H}_{50} \mathrm{O}\right)$. $-{ }^{1} \mathrm{H}$ NMR $\left(500 \mathrm{MHz}, \mathrm{CDCl}_{3}\right.$, $\left.29{ }^{\circ} \mathrm{C}, \mathrm{TMS}\right): \delta=2.38(1 \mathrm{H}, \mathrm{m}, \mathrm{H}-2 \alpha), 2.27(2 \mathrm{H}, \mathrm{m}, \mathrm{H}-2 \beta$, $\mathrm{H}-4), 1.95(1 \mathrm{H}, \mathrm{m}, \mathrm{H}-1 \mathrm{a}), 1.18-1.92$ (m, rest of the protons), $1.16(3 \mathrm{H}, \mathrm{s}, \mathrm{H}-28), 1.03(3 \mathrm{H}, \mathrm{s}, \mathrm{H}-27), 0.99(6 \mathrm{H}, \mathrm{s}$, H-26 \& H-29), 0.93 (3H, s, H-30), 0.86 (3H, d, $J=6.0 \mathrm{~Hz}$, 
$\mathrm{H}-23), 0.85$ (3H, s, H-25), 0.70 (3H, s, H-24). $-{ }^{13} \mathrm{C}$ NMR (125 MHz, $\mathrm{CDCl}_{3}, 29{ }^{\circ} \mathrm{C}$, TMS): $\delta=22.2(\mathrm{C}-1), 41.5$ (C-2), 213.0 (C-3), 58.2 (C-4), 42.1 (C-5), 41.3 (C-6), 18.2 (C-7), 53.1 (C-8), 37.4 (C-9), 59.5 (C-10), 35.6 (C-11), 30.5 (C-12), 39.7 (C-13), 38.3 (C-14), 32.8 (C-15), 36.0 (C-16), 30.0 (C-17), 42.8 (C-18), 35.3 (C-19), 28.2 (C-20), 32.4 (C-21), 39.2 (C-22), 6.8 (C-23), 14.7 (C-24), 17.9 (C-25), 20.3 (C-26), 18.7 (C-27), 32.1 (C-28), 31.8 (C-29), 35.0 (C-30).

\section{Friedlan-3 $\beta$-ol (5) [22]}

By elution with $1 \%$ ethyl acetate in hexane, compound 5 was obtained as colorless needles $(5.5 \mathrm{mg}) .-$ M. p. $=280-$ $283{ }^{\circ} \mathrm{C}$. - MS (EI, $\left.70 \mathrm{eV}\right): m / z=428[\mathrm{M}]^{+}, 413,395$, 289, 275, 248, 220, 206, 149, 137, 125, 69. - HRMS (EI): $\mathrm{m} / \mathrm{z}=428.3996$ (calcd. 428.4018 for $\mathrm{C}_{30} \mathrm{H}_{52} \mathrm{O}$ ). $-{ }^{1} \mathrm{H}$ NMR (500 MHz, $\left.\mathrm{CDCl}_{3}, 29^{\circ} \mathrm{C}, \mathrm{TMS}\right): \delta=3.71$ (1H, br.s, H-3), $1.88(1 \mathrm{H}, \mathrm{dt}, J=9.9,2.0 \mathrm{~Hz}, \mathrm{H}-2 \mathrm{a}), 1.71(1 \mathrm{H}, \mathrm{m}, \mathrm{H}-6 \mathrm{a})$,
$1.56-1.25$ (m, rest of the protons), $1.15(3 \mathrm{H}, \mathrm{s}, \mathrm{H}-30), 0.98$ (3H, s, H-26), 0.97 (6H, s, H-28), 0.96 (6H, s, H-27), 0.94 (3H, s, H-24), 0.92 (3H, s, H-29), 0.91 (3H, d, $J=7.2 \mathrm{~Hz}$, $\mathrm{H}-23), 0.85$ (3H, s, H-25). $-{ }^{13} \mathrm{C}$ NMR (125 MHz, $\mathrm{CDCl}_{3}$, $\left.29{ }^{\circ} \mathrm{C}, \mathrm{TMS}\right): \delta=15.8(\mathrm{C}-1), 35.2(\mathrm{C}-2), 72.7$ (C-3), 49.2 (C-4), 37.1 (C-5), 41.7 (C-6), 17.5 (C-7), 53.2 (C-8), 38.4 (C-9), 61.4 (C-10), 35.3 (C-11), 30.6 (C-12), 37.8 (C-13), 39.7 (C-14), 32.3 (C-15), 36.0 (C-16), 30.0 (C-17), 42.8 (C-18), 35.5 (C-19), 28.2 (C-20), 32.8 (C-21), 39.3 (C-22), 11.6 (C-23), 16.4 (C-24), 18.2 (C-25), 18.6 (C-26), 20.1 (C-27), 32.0 (C-28), 32.1 (C-29), 35.0 (C-30).

\section{Acknowledgements}

We are very much thankful to Mr. Nana Victor of National Herbarium of Yaounde, Cameroon for the identification of plant material. One of us (M. I. A.) kindly acknowledges the enabling role of the Higher Education Commission, Islamabad (Pakistan), and appreciates financial support through "Indigenous Ph. D. 5000 Fellowship Program (Phase II)".
[1] T. N. Misra, S. R. Singh, H. S. Pandey, Y.P. Kohli, International Seminar on Recent Trends in Pharmaceutical Sciences, Ootacamund (Abstract No. 29) 1995.

[2] H. T. Simonsen, J. B. Nordskjold, U. W. Smitt, W. Nyman, P. Palpu, P. Joshi, G. Varughese, J. Ethnopharmacol. 2001, 74, 195-204.

[3] T. Murugesan, K. S. Saravanan, S. Lakshmi, G. Ramya, K. Thenmozhi, Phytomedicine 2001, 8, 472-476.

[4] G. N. Chaturvedi, P. N. Subramaniyam, S. K. Tiwari, K. P. Singh, Ancient Science Life 1984, 3, 216-224.

[5] T. Masuda, S. Yonemori, Y. Oyama, Y. Takeda, T. Tanaka, T. Andoh, A. Shinohara, M. Nakata, J. Agric. Food Chem. 1999, 47, 1749 - 1754.

[6] X.F. Shi, D. J. Du, D.C. Xie, C. Q. Ran, Zhongguo Zhong Yao Za Zhi (China Journal of Chinese Materia Medica), 1993, 18, 687-690.

[7] G. Lammel, H. Rimpler, Z. Naturforsch. 1981, 36c, $708-713$

[8] T. Kanchanapoom, R. Kasai, P. Chumsri, Y. Hiraga, K. Yamasaki, Phytochemistry 2001, 58, 333-336.

[9] R. Pandey, R. K.Verma, S. C. Singh, M. M. Gupta, Phytochemistry 2003, 63, 415-420.

[10] A. U. Rehman, S. Begum, S. Saied, M. I. Choudhary, F. Akhtar, Phytochemistry 1997, 45, 1721 - 1722.
[11] S. Ganapty, D. V. Rao, Indian J. Pharm. Sci. 1985, 47, $167-168$.

[12] R. Roy, V. B. Pandey, Ind. J. Nat. Prod. 1995, 11, $13-$ 14.

[13] T. H. Layne, W. F. Reynolds, S. McLean, W. F. Tinto, Natural Product Commun. 2008, 3, 1787-1792.

[14] R. Roy, V. B. Pandey, Phytochemistry 1994, 37, 1775 1776.

[15] S. Lumbu, C. Hootele, J. Nat. Prod. 1993, 56, $1418-$ 1420 .

[16] M. C. P. Barros, M. A. S. Lima, R. Braz-Filho, E. R. Silveira, Magn. Reson. Chem. 2003, 41, 731-734.

[17] T. Fan, Z. Min, G. Song, M. Iinuma, T. Tanaka, Phytochemistry 1999, 51, $1005-1008$.

[18] X. D. Tian, Z. D. Min, N. Xie, Y. Lei, Z. Y. Tian, Q.T. Zheng, R. N. Xu, T. Tanaka, M. Inuma, M. Mizuno, Chem. Pharn. Bull. 1993, 41, 1415-1417.

[19] S. Liu, H. Zhu, S. Zang, X. Zhang, Q. Yu, L. Xuan, J. Nat. Prod. 2008, 71, 755-759.

[20] V. U. Ahmad, M.S. Ali, Phytochemistry 1991, 30, $4172-4174$.

[21] J. Klass, W. F. Tinto, S. McLean, W. F. Reynolds, J. Nat. Prod. 1992, 55, 1626-1630.

[22] J. K. Kundu, A.S.S. Rouf, M. N. Hossain, C. M. Hasan, A. M. Rashid, Fitoterapia 2000, 71, 577-579. 